\title{
Wenig bekannt: die Sichelzellkrankheit
}

$\mathrm{D}$ ie Sichelzellkrankheit gehört zu den seltenen Erkrankungen, die ein Arzt meist nicht zu sehen bekommt. „Ich spreche von Entwicklungshilfe in Deutschland, denn die Sichelzellkrankheit ist bei uns wenig bekannt, obwohl es sie schon lange gibt", brachte die in einer großen hämatologischen Düsseldorfer Ambulanz tätige Ärztin Dr. Roswitha Dickerhoft ihr Anliegen auf den Punkt. Die Sichelzellkrankheit gehört weltweit zu den häufigsten seltenen Erbkrankheiten.

Aufgrund einer genetischen Veränderung ist die beta-Kette im Hämoglobin so verändert, dass die normalerweise runden, elastischen Erythrozyten ein sichelförmiges Aussehen annehmen. Diese Zellen sind unbeweglicher und sterben früher als gesunde Erythrozyten. Hierdurch kommt es einerseits zur Anämie und andererseits zur Verstopfung der Blutgefäße durch die Sichelzellen mit Gewebeschädigung und oft schweren Schmerzattacken. Betroffen können alle Organsysteme, inklusive das Knochenmark und das ZNS sein. Die bereits

\section{Wann ist ein Kind tot?}

$Z^{\mathrm{u}}$ u den am meisten belastenden Ereignissen in der Pädiatrie gehört die Feststellung des irreversiblen Hirnfunktionsausfalls. „Früher nannte man es Hirntod, heute wird von irreversiblem Hirnfunktionsausfall gesprochen“, erklärte Prof. Dr. Bernhard Roth von der Klinik und Poliklinik für Kinder- und Jugendmedizin des Universitätsklinikums Köln. Um Gewebe und Organe entnehmen zu können, muss der irreversible Hirnfunktionsausfall und Tod eines Menschen festgestellt werden. „Mit der Feststellung des endgültigen, nicht behebbaren Ausfalls der Gesamtfunktion des Großhirns, des Kleinhirns und des Hirnstamms (irreversibeler Hirnfunktionsausfall) ist naturwissenschaftlich-medizinisch der Tod des Menschen festgestellt" [Bundesärztekammer, Vierte Fortschreibung, 2015, S. 2; Dtsch Ärztebl 2015;122: A 1256]. in den ersten Lebensjahren durch verstopfte Blutgefäße geschädigte Milz ist die Ursache für das hohe Infektionsrisiko der Sichelzellpatienten.

Schätzungen gehen von etwa 1.000 Betroffenen aus, wobei nach Dickerhoft die Zahl deutlich steigen wird. „Nicht durch die Zuwanderung der Flüchtlinge, sondern durch Neugeborene, deren Eltern von ihrer Erkrankung nichts wissen“, betonte die Expertin. Heute können über $90 \%$ der Erkrankten das Erwachsenenalter erreichen. Entscheidend hierfür ist die frühe Diagnose und frühzeitige Prävention sowie die fundierte Beratung, Aufklärung und Betreuung von Eltern und Betroffenen. „Wir müssen den Eltern und Kindern bereits früh sagen, dass Sichelzellerkrankte nicht rauchen und kein Cortison bekommen dürfen“, sagte Dickerhoff. Die Therapie ist symptomatisch orientiert, wobei nach Ansicht der Expertin Hydroxycarbamid das einzige Mittel ist, um Schmerzkrisen $\mathrm{zu}$ beherrschen und diesen vorzubeugen. Jeder Patient mit Sichelzellanämie, der

Nach einer von Roth vorgestellten Untersuchung lässt sich die Ätiologie in $44 \%$ der Fälle auf eine traumatische Hirnverletzung zurückführen, in 19\% der Fälle auf Herzkreislaufstillstand, in $10 \%$ auf Meningitis, in $6 \%$ auf intrakranielle Blutungen und zu $21 \%$ auf andere Ätiologien. Befremdlich findet Roth, dass die Leitlinienempfehlungen oft nicht eingehalten werden und bei $19 \%$ der Patienten lediglich eine Untersuchung vorgenommen wurde sowie bei $18 \%$ der obligatorische Apnoe-Test fehlte.

Voraussetzung für eine leitliniengerechte Diagnose eines irreversiblen Hirnfunktionsausfalls ist eine akute schwere primäre Hirnschädigung etwa durch Hirnverletzung, Hirninfarkt oder Hirntumore oder sekundäre Hirnschädigung wie Hypoxie oder Ischämie. Zudem darf keine andere Ursache für die Ausfallsymptome des Gehirns verant-

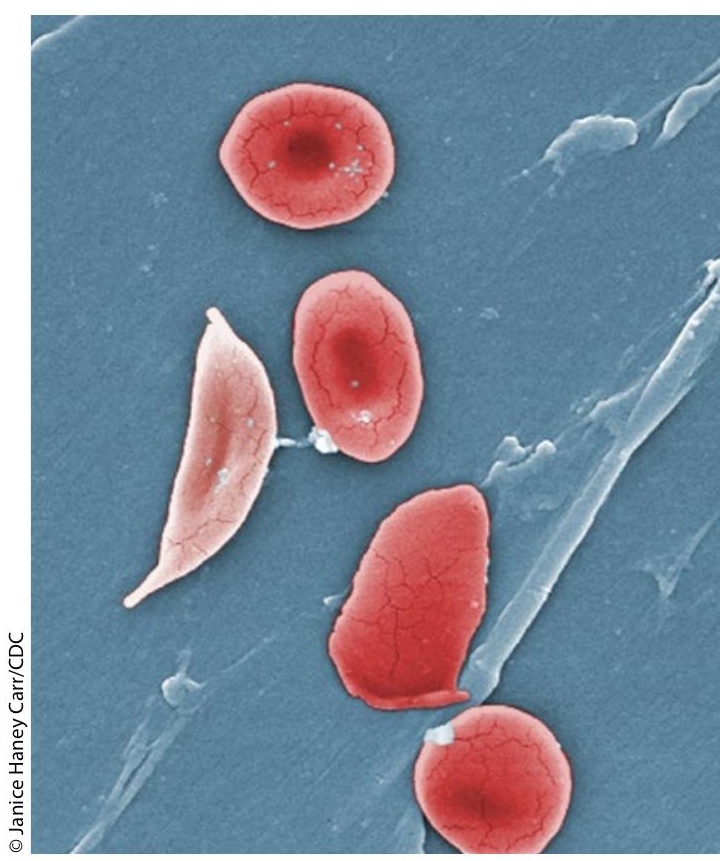

unter Thoraxschmerzen leidet, muss jedoch ins Krankenhaus eingeliefert werden. Dr. Claudia-Viktoria Schwörer

Dickerhoff R. Sichelzellkrankheiten inzwischen ein Alltagsproblem in der Praxis

wortlich sein. Klinisch müssen Koma, Hirnstammareflexie und Apnoe vorliegen. „Der Apnoe-Test ist hierbei ein unverzichtbarer Test“, betonte Roth. Bis zum vollendeten zweiten Lebensjahr sollten ergänzende Untersuchungen wie EEG, frühe akustisch evozierte Potenziale, Doppler und Duplex hinzugezogen werden. Die Leitlinie sieht zwingend eine Wartezeit vor, bevor im letzten Schritt der Irreversibilitätsnachweis mittels klinischer Symptome und ergänzenden Untersuchungen erbracht werden kann. „Es empfiehlt sich bei diesem Prozess die Eltern einzubinden. Der Hirntod eines Kindes wird von diesen wesentlich besser verarbeitet, wenn sie in die Diagnose eingebunden sind“, gab Roth abschließend zu bedenken.

Dr. Claudia-Viktoria Schwörer

Roth B. Leitlinie zur Feststellung des irreversiblen Hirnfunktionsausfalls 